TRANSACTIONS OF THE

AMERICAN MATHEMATICAL SOCIETY

Volume 363, Number 7, July 2011, Pages 3457-3471

S 0002-9947(2011)05401-2

Article electronically published on February 8, 2011

\title{
ON THE BRANCH CURVE OF A GENERAL PROJECTION OF A SURFACE TO A PLANE
}

\author{
C. CILIBERTO AND F. FLAMINI
}

\begin{abstract}
In this paper we prove that the branch curve of a general projection of a surface to the plane is irreducible, with only nodes and cusps. This is a basic result in surface theory, extremely useful in various applications. However, its proof, in this general setting, was so far lacking. Our approach substantially uses a powerful tool from projective differential geometry, i.e., the concept of focal schemes.
\end{abstract}

\section{INTRODUCTION}

A few years ago the first author wrote, in collaboration with R. Miranda and M. Teicher, the paper [8], in which the following theorem, assumed to be well known, was stated and used:

Theorem 1.1. Let $S \subset \mathbb{P}^{r}$ be a smooth, irreducible, projective surface (or a general surface in $\left.\mathbb{P}^{4}\right)$. Then the ramification curve on $S$ of a general projection of $S$ to $\mathbb{P}^{2}$ is smooth and irreducible and the branch curve in the plane is also irreducible and has only nodes and cusps, respectively, corresponding to two simple ramification points and one double ramification point.

This means that the ramification of the general projection morphism $S \rightarrow \mathbb{P}^{2}$ is as simple as possible. This result has been stated, but never proved, by various classical authors (see [11]), and in fact it is extremely useful in various aspects and applications of the theory of surfaces, such as the Hilbert scheme and moduli space computations à la Enriques (see [11], Chapter V, §11), braid monodromy computations (see, e.g., [28, [20, 21]) and in number-theoretical problems concerning algebraic varieties (see, e.g., [12]).

An anonymous referee kindly remarked that there was no proof of Theorem 1.1 in the current literature, despite good evidence for its truth, given the fact that the result holds under the hypothesis that $S$ is the $k$-tuple Veronese embedding of a smooth surface, with $k \geq 2$ (see Kulikov-Kulikov in [22]; in Falting's paper [12] the same result is proved under even stronger hypotheses, verified when $k \geq 5$ ).

Received by the editors February 9, 2009.

2010 Mathematics Subject Classification. Primary 14N05, 14E20, 14E22; Secondary 14J10, $14 \mathrm{E} 05$.

Key words and phrases. Projective surfaces, general projections, coverings, branch curves, singularities.

The authors are members of G.N.S.A.G.A. at I.N.d.A.M. "Francesco Severi". 
The purpose of the present paper is to fill up this annoying gap in the literature, by giving a proof of Theorem 1.1. Actually we will prove the following more general result:

Theorem 1.2. Let $\Sigma \subset \mathbb{P}^{3}$ be a nondegenerate, projective surface, with ordinary singularities. Then the ramification curve of a general projection of $\Sigma$ to a plane from a point $p$ is irreducible, the branch curve is also irreducible and has only nodes and cusps, respectively, corresponding to bitangent, and not tritangent, lines and simple asymptotic tangent lines to $\Sigma$ passing through $p$.

As indicated by Kulikov-Kulikov in [22, Lemma 1.4], Theorem 1.1 (respectively, Theorem 1.2) can be extended to the case in which $S$ (respectively, $\Sigma$ ) has mild singularities (respectively, away from the ordinary singularities); see our Remark 6.1 for this extension.

The proof of Theorem 1.2 consists of a careful study of families of multi-tangent lines to the surface $\Sigma$, which is also present in the approach of [22. The new tool here is the use of some basic techniques in projective-differential geometry, i.e., the theory of focal schemes. This relies on very classical concepts; it has been exposed in a modern setting in [10] and successfully used in several contexts (see, e.g., [5]).

In $₫ 2$ we recall, for the reader's convenience, some basic notions and results about projections of surfaces and the singularities that they produce. In $₫ 3$ we recall the relations between the singularities of the branch curve of a general projection and the tangent lines to the surface passing through the centre of projection (see also 22]). The upshot of the first two sections is to reduce the proof of Theorem 1.1 to the one of Theorem 1.2 and to reduce this, in turn, to the proof that the branch curve has only double points. In $\$ 4$ we recall some generalities about focal schemes. In 95 the focal machinery is applied to prove Proposition [5.1] which is the essential step towards Theorem 1.2 proved in $\$ 6$,

We finish with $\$ 7$ in which we outline the aforementioned application à la Enriques of Theorem 1.1 to the study of the dimension of Hilbert schemes and moduli spaces of surfaces.

In this paper we will work over $\mathbb{C}$.

\section{ORDINARY SINGULARITIES AND PROJECTIONS}

This section is devoted to recalling, for the reader's convenience, some basic facts about general projections to $\mathbb{P}^{3}$ of smooth surfaces sitting in higher dimensional projective spaces.

2.1. Ordinary singularities. We start with a classical definition.

Definition 2.1. An irreducible, projective surface $\Sigma \subset \mathbb{P}^{3}$ is said to have ordinary singularities if its singular locus is either empty or it is a curve $\Gamma$, called the double curve of $\Sigma$, with the following properties:

(1) $\Gamma$ has at most finitely many ordinary triple points, such that the germ of $\Sigma$ there is analytically equivalent to the one of the affine surface in $\mathbb{C}^{3}$ with equation $x y z=0$ at the origin;

(2) every nonsingular point of $\Gamma$ is either a nodal point, i.e., the germ of $\Sigma$ there is analytically equivalent to that of the surface with equation $x^{2}-y^{2}=0$ at the origin, or a pinch point, i.e., the germ of $\Sigma$ there is analytically equivalent to that of the surface with equation $x^{2}-z y^{2}=0$ at the origin; 
(3) for every irreducible component $\Gamma^{\prime}$ of $\Gamma$, the general point of $\Gamma^{\prime}$ is a nodal point of $\Sigma$; in particular, there are only finitely many pinch points for $\Sigma$.

Remark 2.2. Assume $\Sigma \subset \mathbb{P}^{3}$ has ordinary singularities and let $\nu: X \rightarrow \Sigma$ be its normalization. It is immediate to see that $X$ is smooth and the line bundle $\nu^{*}\left(\mathcal{O}_{\mathbb{P}^{3}}(1)\right)$ is ample.

Let $\Delta=\nu^{-1}(\Gamma)$. Then:

(i) $\left.\nu\right|_{\Delta}$ is a generically $2: 1$ covering;

(ii) if $p \in \Gamma$ is a triple point, then $\nu^{-1}(p):=\left\{p_{1}, p_{2}, p_{3}\right\}$, where each $p_{i}$ is a node of $\Delta, 1 \leq i \leq 3$. We will denote by $T$ the set of these nodes, which are the only singular points of $\Delta$;

(iii) if $q \in \Gamma$ is a pinch point, then $q$ is a branch point of $\left.\nu\right|_{\Delta}$, over which $\Delta$ is smooth. We will denote by $\Omega$ the set of corresponding ramification points on $\Delta$.

2.2. Projections. It is a classical fact that surfaces with ordinary singularities occur as general projections in $\mathbb{P}^{3}$ of smooth surfaces in higher dimensional projective spaces. It is useful to recall the basic results on this subject.

Let $S \subset \mathbb{P}^{r}, r \geq 3$, be a smooth, irreducible, nondegenerate projective surface. For any $k<r$, we denote by $\varphi_{k}: S \rightarrow \mathbb{P}^{k}$ the projection of $S$ from a general linear subspace of dimension $r-k-1$ of $\mathbb{P}^{r}$. If $h<k$, we may assume that $\varphi_{h}$ factors through $\varphi_{k}$.

If $r \geq 6$, then $\varphi_{5}: S \rightarrow \mathbb{P}^{5}$ maps $S$ isomorphically to a smooth surface in $\mathbb{P}^{5}$, since the secant variety $\operatorname{Sec}(S)$ does not fill up $\mathbb{P}^{r}$. Projections to $\mathbb{P}^{4}$ and $\mathbb{P}^{3}$ no longer preserve smoothness.

Definition 2.3 (cf. [23, Def. 1]). A nondegenerate, irreducible, projective surface $\Sigma \subset \mathbb{P}^{4}$ is called a general surface of $\mathbb{P}^{4}$ if either $\Sigma$ is smooth or the singularities of $\Sigma$ are at most a finite number of improper double points, i.e., the origin of two smooth branches of $\Sigma$ with independent tangent planes.

The normalization of a general surface in $\mathbb{P}^{4}$ is smooth.

A general projection of a smooth, irreducible, nondegenerate surface $S \subset \mathbb{P}^{r}$, $r \geq 5$, to $\mathbb{P}^{4}$ is a general surface of $\mathbb{P}^{4}$; its improper double points correspond to the intersections of the centre of projection with $\operatorname{Sec}(S)$ and their number is given by the double point formula (see [27, [14]). More precisely, one has (cf. 27] and [23, Thm. 3]):

Theorem 2.4. The only smooth surface $S \subset \mathbb{P}^{5}$ whose general projection to $\mathbb{P}^{4}$ is smooth is the Veronese surface of conics.

This is the same as saying that the Veronese surface of conics is the only smooth defective surface $S$ in $\mathbb{P}^{5}$, i.e., such that $\operatorname{dim}(\operatorname{Sec}(S))<5$.

As for projections to $\mathbb{P}^{3}$, it was classically stated by various authors, such as M. Noether, F. Enriques, etc., that the general projection of a smooth surface to $\mathbb{P}^{3}$ has only ordinary singularities (see, e.g., 11, and 15] for a modern reference). A more precise result is the following (see [23, Thm. 8]):

Theorem 2.5 (The General Projection Theorem). Let $\Sigma$ be a general surface of $\mathbb{P}^{4}$. Then a general projection of $\Sigma$ to $\mathbb{P}^{3}$ has ordinary singularities. 
Though not essential for us, it is worth recalling that, taking the General Projection Theorem for granted, Franchetta proved in [13] the following result (see also [23, Thm. 5]):

Theorem 2.6. Let $\Sigma$ be a general surface of $\mathbb{P}^{4}$. Then the double curve of its general projection to $\mathbb{P}^{3}$ is irreducible, unless $\Sigma$ is the projection of the Veronese surface of conics to $\mathbb{P}^{4}$.

The proof is rather simple if $\Sigma$ is the projection of a smooth surface in $\mathbb{P}^{5}$ (see 24]); otherwise, the argument is quite delicate.

\section{Branch CURVES OF PROJECTIONS}

In this section we recall the relations between the singularities of the branch curve of a general projection and the tangent lines to the surface meeting the centre of projection. Most of this is essentially contained in [22. We dwell on this here in order to make this paper as self-contained as possible.

3.1. Branch curves. Let $\Sigma \subset \mathbb{P}^{3}$ be an irreducible surface with ordinary singularities and let $p \in \mathbb{P}^{3}$ be a general point. Consider the projection $\varphi: \Sigma \rightarrow \mathbb{P}^{2}$ from $p$ to a general plane in $\mathbb{P}^{3}$. If, as above, $\nu: X \rightarrow \Sigma$ is the the normalization, one has the commutative diagram

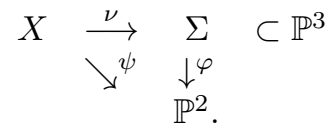

Let $B \subset \mathbb{P}^{2}$ be the branch curve of $\psi$, which in principle may be nonreduced. We will call it the branch curve of $\varphi$ as well. We denote by $R \subset X$ the ramification curve of $\psi$ and by $Z$ its image on $\Sigma$.

Remark 3.1. Note that $Z$ is the residual intersection of $\Sigma$ with its polar with respect to $p$, off the double curve $\Gamma$. The singular locus scheme of a surface $\Sigma \subset \mathbb{P}^{3}$ with equation

$$
f\left(x_{0}, x_{1}, x_{2}, x_{3}\right)=0
$$

is the base locus scheme of the linear system of first polars of $\Sigma$. Recall that the polar of $p=\left[p_{0}, p_{1}, p_{2}, p_{3}\right]$ with respect to $\Sigma$ has the equation

$$
\sum_{i=0}^{3} p_{i} \frac{\partial f}{\partial x_{i}}=0 .
$$

A local computation shows that, if $\Sigma$ has ordinary singularities, the singular locus scheme consists of the double curve $\Gamma$ with an embedded point of length two at each pinch point $z$. This translates the fact that, for any pinch point $z$, all polars are tangent to the plane which is the support of the tangent cone to $\Sigma$ at $z$. By applying Bertini's Theorem we see that $Z$ does not contain any triple point and is smooth at each pinch point. Accordingly, with notation as in Remark 2.2 does not contain any point of $T$ and is smooth at any point of $\Omega$.

Proposition 3.2. In the above setting, the curve $R$ is smooth and irreducible, and therefore $B$ is supported on an irreducible curve. 
Proof. As the centre of projection $p$ varies, the curve $R$ varies in the linear system $\mathcal{R}$ which is the pull-back on $X$ of the linear system cut out on $\Sigma$ by the first polars, off the singular locus.

The base locus scheme of $\mathcal{R}$ consists of $\Omega$; thus the general curve $R \in \mathcal{R}$ is smooth (see Remark 3.1).

Suppose the general curve $R \in \mathcal{R}$ is reducible. By Bertini's theorem, the rational map $\varphi_{\mathcal{R}}$ determined by $\mathcal{R}$ maps $X$ to a curve. On the other hand, the image of this map is the dual of $\Sigma$. This would imply that $\Sigma$ is developable, a contradiction.

Remark 3.3. An alternative argument for the irreducibility of $R$ is the following. Since, as we saw, $R$ is smooth, it suffices to prove that it is connected. Let $H$ be a curve in $\mathcal{L}$. By the Riemann-Hurwitz theorem we have $R \sim K_{X}+3 H$, where $K_{X}$, as usual, denotes a canonical divisor of $X$ and $\sim$ denotes linear equivalence. By adjunction theory (cf., e.g., [17] and $\S 7$ in [9]) one has that $K_{X}+2 H$ is nef and since $H$ is ample, then $R$ is ample; hence it is connected.

Now let $d$ be the degree of $\Sigma$ and $g$ be the geometric genus of its general plane section. Then, by the Riemann-Hurwitz formula, one has $\operatorname{deg}(R)=2(d+g-1)$. If $\varphi$ induces a birational map of $R$ to $B$, then $B$ is reduced and this is also the degree of $B$.

Remark 3.4. If $S \subset \mathbb{P}^{r}, r \geq 5$, is a smooth, irreducible surface, and $\varphi_{2}: S \rightarrow \mathbb{P}^{2}$ is a general projection, we can consider branch and ramification curves of $\varphi_{2}$. In view of the results in $\oint_{2}$, this is a particular case of the previous situation. A similar particular case arises from a general projection to $\mathbb{P}^{2}$ of a general surface in $\mathbb{P}^{4}$.

3.2. Tangent lines. Let $T_{\Sigma}$ be the 3 -dimensional, irreducible subvariety of the Grassmannian $\mathbb{G}(1,3)$ of lines in $\mathbb{P}^{3}$ which is the Zariski closure of the set of all lines tangent to $\Sigma$ at a smooth point. The lines in $T_{\Sigma}$ are called the tangent lines to $\Sigma$.

Given a line $\ell$ in $\mathbb{P}^{3}$, the corresponding point $[\ell] \in \mathbb{G}(1,3)$ sits in $T_{\Sigma}$ if and only if there is a point $z \in \Sigma$ such that $z \in \ell$ and $\ell$ sits in the tangent cone to $\Sigma$ at $z$. In this case we say that $\ell$ is tangent to $\Sigma$ at $z$ and that $z$ is a contact point of $\ell$ with $\Sigma$.

If $\ell \not \subset \Sigma$, we denote by $\ell_{\Sigma}$ the 0 -dimensional scheme cut out by $\ell$ on $\Sigma$ and by $\ell_{X}$ its pull-back to $X$ via $\nu$. Then [ $\left.\ell\right]$ sits in $T_{\Sigma}$ if and only if either $\ell \subset \Sigma$ or $\ell_{X}$ is not reduced.

Let $\ell$ be a line in $\mathbb{P}^{3}$ through the centre of projection $p$. Write $\ell_{X}=n_{1} x_{1}+$ $\ldots+n_{h} x_{h}$, with $n_{1}+\ldots+n_{h}=d$ and $n_{1} \geq \ldots \geq n_{h}$. The line $\ell$ is tangent to $\Sigma$ if and only if there is an $i=1, \ldots, h$ such that $n_{i} \geq 2$, in which case $\ell$ is tangent to $\Sigma$ at $z_{i}=\nu\left(x_{i}\right)$, and $n_{i}-1$ is called the contact order of $\ell$ with the branch of $\Sigma$ corresponding to $x_{i}$ at $z_{i}$.

By the genericity assumption, $p$ does not sit on the developable tangent surface to the double curve $\Gamma$ of $\Sigma$. This implies that there are no pairs of indices $i, j$, with $1 \leq i<j \leq h$ such that $n_{i} \geq 2, n_{j} \geq 2$ and $\nu\left(x_{i}\right)=\nu\left(x_{j}\right)$.

We will say that $\ell$ is $k$-tangent to $\Sigma$ if there are distinct indices $i_{1}, \ldots, i_{k}$ such that $n_{i_{j}} \geq 2$, for all $j=1, \ldots, k$. One says that $\ell$ is an asymptotic tangent line to $\Sigma$ if there is an index $i=1, \ldots, h$ such that $n_{i} \geq 3$, in which case $\ell$ is an asymtpotic tangent line to $\Sigma$ at $z=\nu\left(x_{i}\right)$. The integer $a(\ell)=\sum_{i=1}^{h}\left(n_{i}-2\right)$ is called the asymptotic weight of $\ell$. If $a(\ell)=1$, the line $\ell$ is called a simple asymptotic tangent. 
It is immediate that $y \in \mathbb{P}^{2}$ belongs to $B$ if and only if the line $\ell=\ell_{y}=\langle p, y\rangle$ is tangent to $\Sigma$. If $\ell_{X}=n_{1} x_{1}+\ldots+n_{h} x_{h}$, the integer $b(y)=\sum_{i=1}^{h}\left(n_{i}-1\right)$ is called the branching weight of $y$ and $n_{i}-1$ is the ramification weight $r\left(x_{i}\right)$ of $x_{i}$, $i=1, \ldots, h$. If these weights are 1 or 2 , we will talk about simple or double branch (respectively, ramification) points.

Accordingly $x \in X$ sits on $R$, and then $z=\nu(x)$ sits on $Z$, if and only if the scheme $\ell_{z, X}$ is nonreduced at $x$.

Proposition 3.5. The general tangent line to $\Sigma$ is not $k$-tangent for $k \geq 2$. Hence $\varphi$ induces a birational morphism of $R$ to $B$, which is therefore reduced of degree $2(d+g-1)$.

Proof. The assertion follows by the reflexivity of the general plane section of $\Sigma$.

3.3. More on asymptotic tangent lines. In the above setting, let $x \in X$ and set $z=\nu(x)$. Let $\mathcal{L}$ be the pull-back on $X$ of the linear system of planes in $\mathbb{P}^{3}$. Note that there is a unique curve $C_{x}$ in $\mathcal{L}$ singular at $x$, namely the pull-back to $X$ of the plane section of $\Sigma$ with the tangent plane to the branch through $z$ corresponding to $x$. We will say that $x$ is a planar point of $X$ if $C_{x}$ has a point of multiplicity at least 3 at $x$. If $x$ is a planar point, then all lines through $z$ in the tangent plane to the branch corresponding to $x$ are asymptotic tangent lines.

Lemma 3.6. There are only finitely many planes in $\mathbb{P}^{3}$ cutting $\Sigma$ in a curve with a point of multiplicity $m \geq 3$.

Proof. Suppose the assertion is not true. Then there is a 1-dimensional curve $\left\{C_{t}\right\}_{t \in D}$ in $\mathcal{L}$, parametrized by a disc, whose general member has a point $\left\{x_{t}\right\}_{t \in D}$ of multiplicity $m \geq 3$. The tangent space to this curve at $t=0$ is contained in the set of curves in $\mathcal{L}$ having multiplicity at least $m-1 \geq 2$ at $x_{0}$ (see, e.g., [6] or [26]). Since there is only one such curve, namely $C_{x_{0}}$, we find a contradiction.

By genericity, we may and will assume that the centre of projection stays off the finitely many planes cutting $\Sigma$ in a curve with a point of multiplicity $m \geq 3$.

Suppose $x$ is neither a planar point nor a point in $\Omega$. Then, there are only one or two asymptotic tangent lines through $z$ in the tangent plane to the smooth branch of $\Sigma$ corresponding to $x$ : their directions are the zero locus of the second fundamental form of $\Sigma$ at $x$ (see [16]). In geometric terms, consider the curve $C_{x}$, which has a double point at $x$. The directions of the asymptotic tangent lines at $z=\nu(x)$ are the images via the differential $d \nu_{x}$ of the directions of the lines in the tangent cone to $C_{x}$ at $x$. One says that $x$, or $z=\nu(x)$, is a parabolic point if the tangent cone to $C_{x}$ at $x$ is nonreduced. Then, the corresponding asymptotic tangent line will be called parabolic.

Let $P(S)$ be the Zariski closure of the set of parabolic points of $S$. One has $\operatorname{dim}(P(S)) \leq 1$. Otherwise $\Sigma$ would be a developable surface, i.e., either a cone or the locus of tangent lines to a curve (see [16]). This is not possible, since $\Sigma$ has ordinary singularities.

By the genericity of the centre of projection $p$, we conclude that:

Lemma 3.7. In the above setting, no parabolic asymptotic tangent line contains the centre of projection. 
3.4. Multiple points of the branch curve. The following proposition tells us what are the multiple points of the branch curve. It can be deduced from [18, Theorem IV.1]. We give a short proof for the reader's convenience.

Proposition 3.8. In the above setting, the multiplicity of $B$ at $y$ is the branching weight $b(y)$.

Proof. Let $\ell=\ell_{y}$ and $\ell_{X}=n_{1} x_{1}+\ldots+n_{h} x_{h}$. Let $\pi$ be a general plane through $\ell$ and let $C$ be the pull-back on $X$ of the curve section of $\Sigma$ with $\pi$. Then $C$ is smooth and irreducible of genus $g$. The projection from $p$ induces a morphism $C \rightarrow \mathbb{P}^{1}$ of degree $d$ which has a branch point $y$, corresponding to the line $\ell$ with ramification index $\sum_{i=1}^{h}\left(n_{i}-1\right)$. Bertini's theorem guarantees that the remaining branch points, corresponding to the intersections of $\pi$ with $B$ off $y$, are all simple. Hence their number is $2(d+g-1)-\sum_{i=1}^{h}\left(n_{i}-1\right)=\operatorname{deg}(B)-b(y)$. This proves the assertion.

More specifically:

Proposition 3.9. In the above setting, if B has only double points, then it has only nodes and cusps, respectively, corresponding to bitangent, not tritangent, and simple asymptotic tangent lines containing the centre of projection $p$.

Proof. Let $y$ be a singular point of $B$, which by assumption has multiplicity 2 . Suppose it corresponds to the line $\ell$ through $p$, with $\ell_{X}=n_{1} x_{1}+\ldots+n_{h} x_{h}$. By Proposition 3.8 we have only two possibilities:

(a) $n_{1}=n_{2}=2, n_{3}=\ldots=n_{d-4}=1$; i.e., $\ell$ is a bitangent, not tritangent, line;

(b) $n_{1}=3, n_{2}=\ldots=n_{d-3}=1$; i.e., $\ell$ is a simple asymptotic tangent line.

In case (a), $R$ is smooth at the two points $x_{1}, x_{2}$ over $y$. The lines in the tangent cone to $B$ at $y$ consist of the images, via the projection from $p$, of the two tangent planes $T_{S, x_{i}}$ to $S$ at the branch corresponding to $x_{i}, i=1,2$. We claim that these two planes are distinct, then also their projections from $p$ are distinct, and thus $y$ is a node for $B$.

To prove this, consider the closure $W$ in $\Sigma \times \Sigma$ of the pairs $\left(x_{1}, x_{2}\right)$ of distinct, smooth points, such that $T_{S, x_{1}}=T_{S, x_{2}}$. One has $\operatorname{dim}(W) \leq 1$. In fact the dual $\Sigma^{*}$ of $\Sigma$ is a surface because $\Sigma$ is not a developable surface (see [16]), and the points in $W$ correspond to singular points of $\Sigma^{*}$. Then, by the genericity of $p$, there is no pair of points $\left(x_{1}, x_{2}\right)$ in $W$ such that $p, x_{1}, x_{2}$ are collinear, which proves our claim.

In case (b), we will show, with a direct computation, that $R$ is tangent to $\ell$ at $x:=x_{1}$ and the image of the tangent plane $T_{S, x}$ via the projection from $p$ has intersection multiplicity 3 with $B$ at $y$. This will prove that $y$ is a cusp.

Choosing affine coordinates, we may assume that $p$ is the point at infinity of the $z$-axis, that $\nu(x)$ is the origin, that the tangent plane to $\Sigma$ at $z$ is the plane $y=0$, that the asymptotic tangent lines to $\Sigma$ at the origin are the $z$ and the $y$-axes; note that these asymptotic lines are distinct by Lemma 3.7.

In this coordinate system, $\Sigma$ has an equation of the form

$$
f_{0}(x, z)+y+\sum_{i=2}^{d} y^{i} f_{i}(x, z)=0
$$


where $f_{i}(x, z), i \geq 2$, is a polynomial of degree at most $d-i$ and

$$
f_{0}(x, z)=a x z+a_{1} x^{2}+a_{2} x^{2} z+a_{3} x z^{2}+a_{4} z^{3}+o(3)
$$

with $a \cdot a_{4} \neq 0$.

In this setting, the ideal of $R$ around the origin is generated by the first member of (3.2) and by its derivative with respect to $z$, i.e.,

$$
\frac{\partial f_{0}}{\partial z}(x, z)+\sum_{i=2}^{d} y^{i} \frac{\partial f_{i}}{\partial z}(x, z) .
$$

As we know, $R$ is smooth at the origin (cf. Proposition 3.2). Indeed, the plane $y=0$ is not tangent at the origin to the surface defined as the zero locus of (3.4) because the curve with equation

$$
\frac{\partial f_{0}}{\partial z}(x, z)=0
$$

is not singular at the origin. This is immediate, since the curve in question has the equation

$$
a x+a_{2} x^{2}+2 a_{3} x z+3 a_{4} z^{2}+o(2)=0
$$

with $a \neq 0$. This shows that the $z$-axis $x=y=0$ is tangent to $R$ at the origin.

Note that we can write a local analytic equation of the curve with equation (3.5) as

$$
x=-\frac{3 a_{4}}{a} z^{2}+o(2) .
$$

Next, let us compute the intersection multiplicity of the tangent plane to $\Sigma$ at the origin, with $R$. This amounts to computing the intersection multiplicity at the origin of the two curves

$$
f_{0}(x, z)=0, \quad \frac{\partial f_{0}}{\partial z}(x, z)=0 .
$$

This is the order at 0 of the power series obtained by substituting (3.6) into (3.3), which is clearly 3 . This implies that the line $z=0$ has multiplicity of intersection 3 with $B$ at the origin, proving that $B$ has a cusp there.

\section{FoCAL LOCI}

In this section, we briefly recall some basic definitions and results concerning the so-called focal loci of families of projective varieties. These will be essential in the next section. We follow [5] and [10], inspired in turn by [25].

4.1. The focal machinery. Let $Y$ be a smooth, irreducible, projective variety and let

$$
\begin{array}{ll}
\mathcal{X} & \subset D \times Y \\
\downarrow & \\
D &
\end{array}
$$

be a flat family of closed subschemes of $Y$ parametrized by the base scheme $D$, which we assume to be integral. Denote by

$$
q_{1}: \mathcal{X} \rightarrow D \text { and } q_{2}: D \times Y \rightarrow Y
$$

the natural projections. Set $f:=\left.q_{2}\right|_{\mathcal{X}}$. For every point $z \in D$ we denote by $X_{z}$ the fibre of $q_{1}$ over $z$. 
For any scheme $Z$, set

$$
\mathcal{T}_{Z}:=\mathcal{H o m}\left(\Omega_{Z}^{1}, \mathcal{O}_{Z}\right)
$$

and let

$$
\mathcal{N}:=\mathcal{N}_{\mathcal{X} / B \times Y} \text { and } \mathcal{T}\left(q_{2}\right):=\mathcal{H o m}\left(\Omega_{B \times Y / Y}^{1}, \mathcal{O}_{B \times Y}\right) .
$$

One has the following commutative diagram of sheaves on $\mathcal{X}$ :

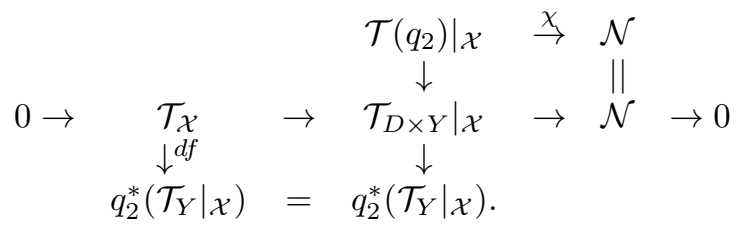

This is called the focal diagram of the family (4.1) (cf. [10, Diagram (3)]).

The map $\chi$ is defined by the commutative diagram and is called the global characteristic map of the family (4.1). From the focal diagram (4.2), one sees that $\operatorname{ker}(\chi)=\operatorname{ker}(d f)$. We denote by $\mathcal{F}$ this sheaf. We will mainly consider the case in which $f: \mathcal{X} \rightarrow Y$ is dominant and generically finite, so that $\mathcal{F}$ is a torsion sheaf, which we call the focal sheaf of the family (4.1). Its support $\mathcal{F}(\mathcal{X})$ is called the focal scheme of the family, and $\operatorname{dim}(\mathcal{F}(\mathcal{X}))<\operatorname{dim}(\mathcal{X})$. If $z \in D$ is a point, we denote by $\mathcal{F}\left(X_{z}\right)$ the intersection of the focal scheme with $X_{z}$.

From the focal diagram (4.2), one can think of $\mathcal{F}(\mathcal{X})$ as the set of ramification points of the map $f$. We denote by $L(\mathcal{X})$ its image via $f$, i.e., the set of branch points of $f$.

4.2. Filling families of linear spaces. The situation to have in mind for our applications is the following: $Y=\mathbb{P}^{r}$ and $\mathcal{X}$ is a family of $k$-dimensional linear subspaces of $\mathbb{P}^{r}$ such that $\operatorname{dim}(D)=r-k$ and $f: \mathcal{X} \rightarrow \mathbb{P}^{r}$ is dominant. This is called a filling family of linear subspaces of $\mathbb{P}^{r}$. For example, $D$ could be an $(r-k)-$ dimensional subscheme of the Grassmannian $\mathbb{G}(k, r)$, with the filling property.

Since we will work at the general point of $D$, and since $D$ is integral, we may and will assume that $D$ is smooth.

Proposition 4.1. If (4.1) is a filling family of $k$-dimensional linear subspaces of $\mathbb{P}^{r}$ and if $z$ is a general point of $D$, then $\mathcal{F}\left(X_{z}\right)$ is a hypersurface of degree $r-k$ in $X_{z} \simeq \mathbb{P}^{k}$.

Proof. If one restricts the global characteristic map $\chi$ to the fibre $X_{z}$, this reduces to

$$
\mathcal{O}_{X_{z}}^{\oplus(r-k)} \cong T_{D, z} \otimes \mathcal{O}_{X_{z}} \stackrel{\chi_{z}}{\longrightarrow} \mathcal{N}_{X_{z} / \mathbb{P}^{r}} \cong \mathcal{O}_{X_{z}}(1)^{\oplus(r-k)} .
$$

In particular, for any $z \in D$, the map $\chi_{z}$ can be viewed as a square matrix $A_{z}$ of size $r-k$, with linear entries. Thus, $\mathcal{F}\left(X_{z}\right)$ is defined by the equation $\operatorname{det}\left(A_{z}\right)=0$ and the assertion follows. Note that $\operatorname{det}\left(A_{z}\right)$ cannot be identically zero, because $\operatorname{dim}(\mathcal{F}(\mathcal{X}))<\operatorname{dim}(\mathcal{X})$, and therefore also $\operatorname{dim}\left(\mathcal{F}\left(X_{z}\right)\right)<\operatorname{dim}\left(X_{z}\right)=k$.

4.3. Filling families of lines in $\mathbb{P}^{3}$. More specifically, we will consider filling families of lines in $\mathbb{P}^{3}$. In this case on the general line of the family there are two foci, which can either be distinct or can be just one with multiplicity 2 . It is useful to recall how the equation of the foci can be computed on the general line $\ell$ of the family $\mathcal{X}$. 
Since the problem is local, we may assume the family to be parametrized by a bidisc $D$. More precisely, if $z=(u, v)$ is a point of $D$, we may assume that the line $\ell_{z}$ is described as the intersection of the two planes with equation

$$
a(z) \times x=b(z) \times x=0,
$$

with

$$
\begin{aligned}
a(z) & =\left(a_{0}(z), a_{1}(z), a_{2}(z), a_{3}(z)\right), \\
b(z) & =\left(b_{0}(z), b_{1}(z), b_{2}(z), b_{3}(z)\right), \\
x & =\left(x_{0}, x_{1}, x_{2}, x_{3}\right) .
\end{aligned}
$$

We may write $a, b, a_{i}, b_{i}$ rather that $a(z), b(z), a_{i}(z), b_{i}(z)$. We will denote with lower case indices the derivatives with respect to the variables $u, v$, i.e. $a_{u}=\frac{\partial a}{\partial u}$, $a_{i, u}=\frac{\partial a_{i}}{\partial u}$, etc.

In this setting, the characteristic map can be described by looking at (4.3). One sees that the equation of the focal locus on $\ell_{z}$ is

$$
\operatorname{det}\left(\begin{array}{cc}
a_{u} \times x & a_{v} \times x \\
b_{u} \times x & b_{v} \times x
\end{array}\right)=0
$$

modulo (4.4).

\section{Filling FAMilies of tANGENT Lines tO A SURFACE}

In this section, using focal techniques, we prove a proposition which is an essential tool for the proof of Theorem 1.1. This proposition was certainly known to the classical algebraic and projective-differential geometers. Since however we do not have a suitable reference for it, we give its complete proof here.

Proposition 5.1. Let $\mathcal{X}$ be a filling family of lines in $\mathbb{P}^{3}$. Assume that its general member $\ell$ is tangent to a nondevelopable surface $\Sigma$ at a general point $p$ of it. Then:

(a) $p$ is a focus on $\ell$;

(b) the contact order of $\ell$ with $\Sigma$ at $p$ is at most 2 ;

(c) if the contact order of $\ell$ with $\Sigma$ at $p$ is 2 , then $p$ is a focus with multiplicity two on $\ell$.

Proof. The question being local, we may assume that $\mathcal{X}$ is given around $\ell$ as follows. Let $\Sigma$ be locally parametrized around $p$ as $p=p(u, v)$, with $z=(u, v) \in D$, where $D$ is a bidisc. Then $\ell_{z}$ is defined by the equations (4.4), where we may assume that the plane $a \times x=0$ is tangent to $\Sigma$ at $p$; i.e., one has

$$
a \times p=a \times p_{u}=a \times p_{v}=0 .
$$

By differentiating the first relation in (5.1) and taking into account the other two, we find

$$
a_{u} \times p=a_{v} \times p=0 .
$$

Taking into account equation (4.5), (a) immediately follows.

Before proceeding, note that the dual variety $\Sigma^{*}$ of $\Sigma$ is a surface, since we are assuming that $\Sigma$ is not developable (see [16]). This implies that $a, a_{u}$, and $a_{v}$ are linearly independent. 
Assume now that $\ell$ is an asymptotic tangent line to $\Sigma$ at $p$. We may suppose that $\ell=\ell_{z}$ has the tangent direction of the vector $p_{u}$ at $p$. This translates into the relation

$$
a \times p_{u u}=0
$$

and $\ell_{z}$ is parametrically described by

$$
x=x_{0} p+x_{1} p_{u} .
$$

By differentiating the second equation in (5.1) and taking into account (5.3), we find

$$
a_{u} \times p_{u}=0 .
$$

Hence the plane $a_{u} \times x=0$ is distinct from the tangent plane $a \times x=0$ and contains the line $\ell$. So $\ell$ is defined by the equations

$$
a \times x=a_{u} \times x=0
$$

and the equation (4.5) of the focal locus becomes

$$
\operatorname{det}\left(\begin{array}{cc}
a_{u} \times x & a_{v} \times x \\
a_{u u} \times x & a_{u v} \times x
\end{array}\right)=0 .
$$

By differentiating the first equation in (5.2) and considering (5.5), we get

$$
a_{u u} \times p=0 .
$$

Substituting (5.4) into (5.6), and taking into account (5.2), (5.5) and (5.7), we find the equation

$$
x_{1}^{2}\left(a_{v} \times p_{u}\right)\left(a_{u u} \times p_{u}\right)=0,
$$

which, by the filling property, is not identically zero (see Proposition 4.1).

Note that $a_{v} \times p_{u} \neq 0$, because, as we saw, $a_{v}$ is linearly independent from $a$ and $a_{u}$. In addition we have $a_{u u} \times p_{u} \neq 0$. On the other hand, by differentiating (5.3) and (5.5), we see that

$$
a_{u u} \times p_{u}=a \times p_{u u u} .
$$

Thus one has $a \times p_{\text {uuu }} \neq 0$, which proves (b). Summing up, the equation (5.8) becomes $x_{1}^{2}=0$, proving (c).

Remark 5.2. As a consequence of Proposition 5.1, there is no nondevelopable surface $\Sigma$ in $\mathbb{P}^{3}$ having a 2 -dimensional family $\mathcal{X}$ of nonsimple asymptotic tangent lines. The same holds if $\Sigma$ is developable but not a plane. We do not dwell on this now.

Conversely, if $\mathcal{X}$ is a filling family of lines in $\mathbb{P}^{3}$, which does not have fundamental points, i.e., points $p \in \mathbb{P}^{3}$ contained in infinitely many lines of $\mathcal{X}$, then the focal locus of $\mathcal{X}$ in $\mathbb{P}^{3}$ is a, may be reducible, surface $\Sigma$ and $\mathcal{X}$ is formed by lines which are either bitangents or asymptotic tangents to $\Sigma$. We do not dwell on this as well.

\section{THE PROOFs OF THE MAIN THEOREMS}

We are now in a position to give the:

Proof of Theorem 1.2, By Proposition 3.9, it suffices to prove that $B$ has only double points. In view of Proposition 3.8, one has to show that there is no filling 
family of lines whose general member $\ell$ is such that $\ell_{X}=n_{1} x_{1}+\ldots+n_{h} x_{h}$ with $\sum_{i=1}^{h}\left(n_{i}-1\right) \geq 3$. This is ensured by $\$ 4.2$ and by Proposition 5.1 .

Finally, we have the:

Proof of Theorem [1.1. It follows from Theorem 1.2 and from the General Projection Theorem 2.5 .

Remark 6.1. Suppose that $\Sigma$ has, off the ordinary singularity locus, a finite number of further double points where the germ of $\Sigma$ is analytically equivalent to that of an affine surface in $\mathbb{C}^{3}$ with equation $z^{2}=h(x, y)$, at the origin, where $h(x, y)=0$ is a curve singular at the origin. As in 22, in particular see Lemma 1.4, one proves that the branch curve $B$ of a general projection of $\Sigma$ to a plane has again only nodes and cusps besides the singularities arising from the projections of the aforementioned double points, where the singularity of $B$ is locally analytically equivalent to that of the curve $h(x, y)=0$. This extension of Theorem 1.2 implies an obvious analogous extension of Theorem 1.1. Note however that the irreducibility statement about branch and ramification curves fails in this situation. A counterexample is given by the branch curve of a general projection of a quadric cone to the plane, which consists of the union of two lines. However, this is the only case in which this happens. Indeed, with the same argument as in the proof of Proposition 3.2. one shows that $R$ is reducible only if $\Sigma$ is developable. This, in the present case, can happen only if $\Sigma$ is a quadric cone.

\section{Applications to Hilbert scheme dimension COMPUTATions}

As mentioned in the introduction, Theorem 1.1 has important applications in the theory of surfaces. In this section we recall one, namely Enrique's approach to the computation of the dimension of Hilbert schemes and moduli spaces of surfaces (see [1]).

Let $S \subset \mathbb{P}^{r}$ and $\varphi:=\varphi_{2}: S \rightarrow \mathbb{P}^{2}$ be as usual. One has the exact sequence

$$
0 \rightarrow T_{S} \stackrel{d \varphi}{\longrightarrow} \varphi^{*}\left(T_{\mathbb{P}^{2}}\right) \rightarrow \mathcal{N}_{\varphi} \rightarrow 0
$$

defining $\mathcal{N}_{\varphi}$, which is called the normal sheaf to the map $\varphi$, fitting also in the so-called Rohn exact sequence

$$
0 \rightarrow \bigoplus_{i=1}^{r-2} \mathcal{O}_{S}(H) \rightarrow \mathcal{N}_{S / \mathbb{P}^{r}} \rightarrow \mathcal{N}_{\varphi} \rightarrow 0
$$

(see, e.g., [7, p. 358, formula (2.2)]).

The sheaf $\mathcal{N}_{\varphi}$ has torsion, being supported on $R \subset S$, the ramification locus of $\varphi$. The morphism $\phi:=\left.\varphi\right|_{R}: R \rightarrow B$ is birational onto the branch curve, which has only nodes and cusps as singularities by Theorem 1.1. and

$$
\operatorname{deg}(R)=\operatorname{deg}(B)=2(d+g-1)
$$

Let $G \subset R$ be the divisor formed by all points $p$ such that $\phi(p)$ is a cusp of $B$, each counted with multiplicity one, and let $i: R \hookrightarrow S$ be the inclusion of $R$ in $S$. By the exact sequence (7.1) and the analogous sequence for the map $\phi$, we get the 
commutative diagram

$$
\begin{aligned}
& 0 \\
& \downarrow \\
& \mathcal{G} \cong \mathcal{O}_{G} \\
& \downarrow \\
& \begin{array}{ccccccc}
0 \rightarrow & T_{R} & \stackrel{d \phi}{\longrightarrow} & \phi^{*}\left(T_{\mathbb{P}^{2}}\right) & \rightarrow & \mathcal{N}_{\phi} & \rightarrow 0 \\
\downarrow & & \| & & \downarrow & \\
& i^{*}\left(T_{S}\right) & \stackrel{d \varphi}{\longrightarrow} & \phi^{*}\left(T_{\mathbb{P}^{2}}\right) & \rightarrow & i^{*}\left(\mathcal{N}_{\varphi}\right) & \rightarrow 0
\end{array} \\
& \downarrow \\
& 0 \text {. }
\end{aligned}
$$

Now consider the following diagram (see [1, p. 24]):

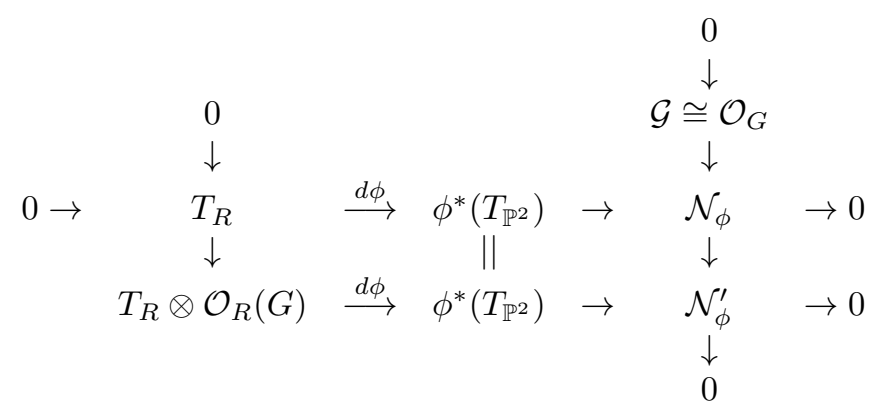

where $\mathcal{N}_{\phi}^{\prime}$ is a line bundle on $R$. Note that these two diagrams imply that

$$
h^{j}\left(S, \mathcal{N}_{\varphi}\right)=h^{j}\left(R, i^{*}\left(\mathcal{N}_{\varphi}\right)\right)=h^{j}\left(R, \mathcal{N}_{\phi}^{\prime}\right), \quad j=0,1,2 .
$$

One has

$\operatorname{deg}\left(\mathcal{N}_{\phi}\right)=\operatorname{deg}\left(\phi^{*}\left(T_{\mathbb{P}^{2}}\right)\right)-\operatorname{deg}\left(T_{R}\right)=3 \operatorname{deg}(R)+\operatorname{deg}\left(K_{R}\right)=6(d+g-1)+\operatorname{deg}\left(K_{R}\right)$.

The classical formula for the number of cusps of the branch curve, in case this has only nodes and cusps, gives

$$
\operatorname{deg}(Z)=3\left(d+K_{S}^{2}-4 \chi\left(\mathcal{O}_{S}\right)+6(g-1)\right)
$$

(cf. formulas (a), (b) and (d) in [8, Prop. 2.6]; cf. standard references as [11, p. 182] and [19]).

Thus

$$
\operatorname{deg}\left(\mathcal{N}_{\phi}^{\prime}\right)=\operatorname{deg}\left(\mathcal{N}_{\phi}\right)-\operatorname{deg}(Z)=3\left(d-K_{S}^{2}-4(g-1)+4 \chi\left(\mathcal{O}_{S}\right)\right)+\operatorname{deg}\left(K_{R}\right) .
$$

Since

$$
p_{a}(R)=9(g-1)+K_{S}^{2}+1
$$

one has

$$
h^{0}\left(S, \mathcal{N}_{\varphi}\right)=h^{0}\left(R, \mathcal{N}_{\phi}^{\prime}\right)=3 d-3(g-1)-2 K_{S}^{2}+12 \chi\left(\mathcal{O}_{S}\right)+h,
$$

where $h=h^{1}\left(R, \mathcal{N}_{\phi}^{\prime}\right)=h^{1}\left(S, \mathcal{N}_{\varphi}\right)$. Note that $\mathcal{N}_{\phi}^{\prime}$ is nonspecial, hence $h=0$, if

$$
d-K_{S}^{2}-4(g-1)+4 \chi\left(\mathcal{O}_{S}\right)>0 .
$$


Otherwise, in case $\mathcal{N}_{\phi}^{\prime}$ is special, note that $R$ is not hyperelliptic, since $\mathcal{O}_{R}(H)$ is clearly special. Then, taking into account (7.6), one has

$$
h \leq \frac{3}{2}\left(K_{S}^{2}-d\right)+6\left(g-1-\chi\left(\mathcal{O}_{S}\right)\right)+\frac{1}{2},
$$

by Clifford's theorem. More precisely, let

$$
h^{1}\left(S, \mathcal{O}_{S}(H)\right)=h(S)
$$

be the speciality of $S$.

From (7.2), (7.3), (7.4), (7.5), (7.7) and (7.9) we conclude that:

Theorem 7.1. In the above setting, one has

$$
\begin{aligned}
h^{0}\left(S, \mathcal{N}_{S / \mathbb{P}^{r}}\right) & \leq(r-2)(r+1)+h^{0}\left(R, \mathcal{N}_{\phi}^{\prime}\right) \\
& =(r-2)(r+1)+3 d-3(g-1)-2 K_{S}^{2}+12 \chi\left(\mathcal{O}_{S}\right)+h .
\end{aligned}
$$

If $\mathcal{N}_{\phi}^{\prime}$ is nonspecial (in particular if (7.8) holds) one has:

(i) $h^{1}\left(S, \mathcal{N}_{S / \mathbb{P}^{r}}\right) \leq(r-2) h(S)$,

(ii) $h^{2}\left(S, \mathcal{N}_{S / \mathbb{P}^{r}}\right)=(r-2) h^{2}\left(\mathcal{O}_{S}(H)\right)$.

The above inequalities become equalities if and only if the map $H^{0}\left(\mathcal{N}_{S / \mathbb{P} r}\right) \rightarrow$ $H^{0}\left(\mathcal{N}_{\varphi}\right)$ arising from (7.2) is surjective. This is the case if $h(S)=0$.

If $\mathcal{N}_{\phi}^{\prime}$ is special, one has

$$
h^{0}\left(S, \mathcal{N}_{S / \mathbb{P}^{r}}\right) \leq\left(r^{2}-r-1\right)+\frac{1}{2}\left(3 d-K_{S}^{2}-1\right)+3(g-1)+6 \chi\left(\mathcal{O}_{S}\right) .
$$

Remark 7.2. Suppose that $H \sim K_{S}$, i.e., that the canonical system is very ample. Let $q(S)=h^{1}\left(S, \mathcal{O}_{S}\right)$ be, as usual, the irregularity of $S$. The above theorem implies that the number $M(S)$ of moduli of $S$ is bounded above by $4 K_{S}^{2}+3 \chi\left(\mathcal{O}_{S}\right)-3 q(S)+4$, if $h>0$, and by $12 \chi\left(\mathcal{O}_{S}\right)-3 p_{g}-1-2 K_{S}^{2}$. In the latter case, since $K_{S}^{2} \geq 3 p_{g}-7$ by Castelnuovo's inequality (see, e.g., [2]), one has $M(S) \leq 3 p_{g}-12 q+25$. For the problem of finding a good upper bound for the number of moduli of a surface, see [3], 4].

\section{ACKNOWLEDGMENTS}

We wish to thank Michael Friedman and Mina Teicher for reminding us of reference [12, and Jonathan Wahl for reminding us of reference [18.

\section{REFERENCES}

1. E. Arbarello, M. Cornalba, Su una congettura di Petri, Comment. Math. Helvetici, 56(1981), 1-38. MR615613 (82k:14029)

2. W. Barth, K. Hulek, C. Peters, A. Van de Ven, Compact Complex Surfaces, Ergebnisse der Mathematik und ihrer Grenzgebiete. 3. Folge. A Series of Modern Surveys in Mathematics, 4. Springer-Verlag, Berlin, 2004. MR2030225 (2004m:14070)

3. F. Catanese, Moduli of surfaces of general type, in "Algebraic Geometry - Open Problems", Proceedings of the Ravello Conference, 1982, Springer Lecture Notes in Math., 997 (1983), 90-112. MR714742 (84k:14026)

4. F. Catanese, On the moduli spaces of surfaces of general type, Jour. of Diff. Geom., 19 (1984), no. 2, 483-515. MR755236 (86h:14031)

5. L. Chiantini, C. Ciliberto, A few remarks on the lifting problems, Journées de géométrie algébrique d'Orsay, Astérisque, 218 (1993), 95-109. MR.1265310(95c:14072)

6. L. Chiantini, C. Ciliberto, Weakly defective varieties, Transactions Amer. Math. Soc., 354 (2001), 151-178. MR 1859030(2003b:14063) 
7. C. Ciliberto, On the Hilbert scheme of curves of maximal genus in a projective space, Math. Z., 194 (1987), 351-363. MR879937 (88c:14036)

8. C. Ciliberto, R. Miranda, M. Teicher, Pillow degenerations of $K 3$ surfaces, Applications of algebraic geometry to coding theory, physics and computations, (Eilat 2001), 53-63, NATO Sci. Ser. II Math. Phys. Chem., 36, Kluwer Acad. Publ., Dordrecht, 2001. MR.1866895 (2002h:14063)

9. C. Ciliberto, F. Russo, Varieties with minimal secant degree and linear systems of maximal dimension on surfaces, Adv. Math., 200 (2006), 1-50. MR2199628 (2007d:14097)

10. C. Ciliberto, E. Sernesi, Singularities of the theta divisor and congruences of planes, J. Algebraic Geom., 1 (1992), 231-250. MR.1144438(92j:14034)

11. F. Enriques, Le superficie algebriche, Zanichelli, Bologna, 1949. MR0031770 (11:202b)

12. G. Faltings, A new application of Diophantine approximations. A panorama of number theory or the view from Baker's garden (Zürich, 1999), 231-246, Cambridge Univ. Press, Cambridge, 2002. MR:1975455 (2004b:11100)

13. A. Franchetta, Sulla curva doppia della proiezione della superficie generale dell' $S_{4}$, da un punto generico su un $S_{3}$, Rend. Acc. d'Italia Ser. VII 2 (1940), 282-288 and Lincei - Rend. Sc. fis. mat. nat. 2 (1947), 276-279. MR0022696 (9:248b)

14. W. Fulton, Intersection theory, Springer Verlag, 1998. MR1644323 (99d:14003)

15. P. Griffiths, J. Harris, Principles of algebraic geometry, Pure and Applied Mathematics. Wiley-Interscience, John Wiley and Sons, New York, 1978. MR.507725 (80b:14001)

16. P. Griffiths, J. Harris, Algebraic geometry and local differential geometry, Ann. Sci. Ecole Norm. Sup., 12 (1979), 355-432. MR.559347 (81k:53004)

17. P. Ionescu, Generalized adjunction and applications, Math. Proc. Camb. Phil. Soc, 99 (1986), 467-472. MR830359 (87e:14031)

18. B. Iversen, Numerical invariants and multiple planes, Amer. J. of Math., 92 (4) (1970), 968996. MR0296074 (45:5135)

19. B. Iversen, Critical points of an algebraic function, Invent. Math., 12 (1971), 210-224. MR 0342512 (49:7258)

20. V. Kulikov, Generic coverings of the plane and braid monodromy invariants, The Fano Conference, 533-558, Univ. Torino, Turin, 2004. MR2112590 (2005j:14050)

21. V. Kulikov, Hurwitz curves, Russian Math. Surveys, 62 (6), 1043-1119. MR.2382475 (2009a:14037)

22. V. Kulikov, V. S. Kulikov, Generic coverings of the plane with A-D-E-singularities, Izvestiya: Mathematics, 64 (6), 1153-1195. MR1817250(2002c:14058)

23. E. Mezzetti, D. Portelli, A tour through some classical theorems on algebraic surfaces. An. Stiint. Univ. Ovidius Constanta Ser. Mat., 5 (1997), no. 2, 51-78. (cf. also http://it.wikipedia.org/wiki/Teorema_di_Kronecker-Castelnuovo) MR.1614780 (98m:14036)

24. B. Moishezon, Complex surfaces and connected sums of complex projective planes. Lecture Notes in Mathematics, 603. Springer-Verlag, New York, 1977. MR0491730 (58:10931)

25. C. Segre, Sui fuochi di $2^{\circ}$ ordine dei sistemi infiniti di piani e sulle curve iperspaziali con una doppia infinità di piani plurisecanti, Atti R. Accad. Lincei, (5) 30 (1921), 67-71.

26. E. Sernesi, Deformations of Algebraic Schemes, Grundlehren der Mathematischen Wissenschaften 334. Springer-Verlag, Berlin, 2006. MR2247603 (2008e:14011)

27. F. Severi, Intorno ai punti doppi impropri di una superficie generale dello spazio a quattro dimensioni, e ai suoi punti tripli apparenti, Rend. Circolo Matematico di Palermo, 15 (1901), 33-51; also in Opere Matematiche, I, VI, 14-30.

28. M. Teicher, Braid Group, Algebraic Surfaces and Fundamental Groups of complements of Branch Curves, Algebraic Geometry - Santa Cruz 1995, 127-150, Proc. Sympos. Pure Math, 62, part 1, Amer. Math. Soc., Providence, RI, 1997. MR.1492521 (99a:14046)

Dipartimento di Matematica, Università degli Studi di Roma Tor Vergata, Via della Ricerca Scientifica, 00133 Roma, Italy

E-mail address: cilibert@mat.uniroma2.it

Dipartimento di Matematica, Università degli Studi di Roma Tor Vergata, Via della Ricerca Scientifica, 00133 Roma, Italy

E-mail address: flamini@mat.uniroma2.it 